\title{
Fast tomographic inspection of cylindrical objects
}

\author{
Wannes Goethals · Marjolein Heyndrickx · \\ Matthieu Boone
}

Received: 18/09/2019 / Accepted: date

\begin{abstract}
This paper presents a method for improved analysis of objects with an axial symmetry using X-ray Computed Tomography (CT). A cylindrical coordinate system about an axis fixed to the object's center of symmetry forms the most natural base to analyze characteristics of those objects that exhibit axial symmetry, as is often the case for industrial parts. As the sampling grid in the proposed methodology is chosen in reference to the object, down-sampling may be applied in directions where no good resolution is required, to reduce reconstruction time without reducing quality. This is very valuable for in-line applications and fast quality inspection. In combination with algebraic reconstruction algorithms, it permits the use of a pre-computed initial volume perfectly suited to fit a series of scans where same-type objects can have different positions and orientations. Weighted back-projection can also be included when some regions are more likely subject to change, to improve reconstruction stability and defect sensitivity.

In this paper, an innovative projection-based pose estimation technique is presented and validated on realistic simulated projections that resemble a setup with high-throughput conditions. Four cylindrical reconstruction strategies are compared to observe the influence of using an initial volume or weighted back-projection on tomographic inspection of medical devices. Finally, the effect of down-sampling the cylindrical reconstruction is analysed on phantoms with varying spatial frequency.
\end{abstract}

Keywords X-rays, tomography, 3D inspection, in-line, cylindrical, pose estimation

W. Goethals

UGCT-Department of Physics and Astronomy, Proeftuinstraat 86, 9000 Ghent, Belgium

E-mail: wannes.goethals@ugent.be

M. Heyndrickx

E-mail: marjolein.heyndrickx@ugent.be

M. Boone

E-mail: matthieu.boone@ugent.be 


\section{Introduction}

Non-destructive evaluation, either in-line or on random test samples, is paramount in a modern production environment to detect internal faults at an early stage. For assembled products, a common fault case is the situation where a component is missing in the final product. These components are often concealed in the assembly, hindering visual inspection. X-ray based imaging may offer better insight on manufacturing quality.

For in-line inspection, X-ray transmission scanning is usually applied to make 2D projection images. Common examples can be found in defect detection [1-3] or material identification [4]. On the other hand, the more intensive 3D imaging by X-ray CT is mainly used off-line. The first industrial applications of CT can be found since the 1980s in the field of material analysis, e.g. observing inner structure and processes or locating internal defects. More recently, CT scanners are being used dedicated to metrology for dimensional quality control. High importance is given in this case to accuracy, traceability to the unit of length and measurement uncertainty [5-7]. New developments in CT technology, driven by research at synchrotron facilities and medical imaging, allow the technology to be applied in an automated production environment too [8, 9].

As the industry is gradually becoming aware of the options that X-ray CT offers as a nondestructive 3D inspection tool, this leads to the demand for systems specifically designed for scanning sequences of same-type objects in a high throughput environment. The challenges lie in the fact that a variety of factors restrict the number of projections to as low as technically feasible. The solutions can be found in more advanced reconstruction techniques like discrete reconstruction [10] or neural-network based solutions [11]. Even though iterative reconstructions cannot compete with the analytic counterparts in terms of reconstruction time for standard cone-beam geometry, they allow making fewer projections with still reasonable results by incorporating prior knowledge. This reduces the scan time and the absorbed radiation dose per object.

Previous studies have shown that iterative reconstruction schemes using cylindrical coordinates can alleviate memory requirements and be less subject to noise than Filtered BackProjection (FBP) algorithms [12]. A potential downside was identified in Jian et al. [13] , which reports potential loss in resolution due to non-uniform sampling. In these studies, the cylindrical coordinate axis is positioned along the vertical axis of the rotation stage.

In this work the specific choice is made not to fix the reconstruction axis to the vertical rotation axis, but to attach the coordinate system to the detected symmetry axis of the scanned object. In an industrial environment, misalignment of this axis w.r.t. the measurement system is the rule rather than the exception, so it warrants attention. This tracking reconstruction is computationally more intensive, as it excludes the reported acceleration of the algorithms mentioned before. It will however be highly beneficial in both the reconstruction and analysis phase. A point-tracking technique, presented in part 2.1 has been developed to automatically derive the $3 \mathrm{D}$ pose of the scanned object from the projection images. It was tested extensively using CAD-based phantom simulations. Given the simple symmetry no extension to more demanding deep learning techniques [14, 15] should be made, which are more fit for samples with lower symmetry. Using this prior knowledge on the $3 \mathrm{D}$ pose, the algebraic reconstruction is improved by including an initial solution that automatically has a correct orientation and that is adapted to the more convenient cylindrical coordinate system. 
Within the same coordinates, regions can be marked to have a high or low probability of change to improve reconstruction stability (section 2.2). The effect of aimed resampling is demonstrated on simulated data in section 2.3 and improved reconstruction algorithms in cylindrical coordinates are applied on a large series of scans in 2.4

\section{Methods and materials}

Two techniques are proposed to improve CT reconstruction of misaligned cylindrical objects.

1. To detect the cylinder orientation from the projection data, a point tracking algorithm is made for general trajectories.

2. The cylindrical reconstruction is built as an extension of a regular Cartesian Simultaneous Algebraic Reconstruction Technique (SART).

The object's pose, illustrated in figure 1. can be captured in six degrees of freedom. Three intrinsic $z x z$ Euler angles $(\alpha, \beta, \gamma)$ are captured in the rotation matrix $R$, while $T$ translates the object along the three spatial coordinates:

$$
X^{\prime}=R^{-1}(X-T)
$$

After the alignment from $X=(x, y, z)$ to $X^{\prime}=\left(x^{\prime}, y^{\prime}, z^{\prime}\right)$ in equation (1), a transformation to cylindrical coordinates around the $\mathrm{z}^{\prime}$-axis is performed on this oriented set of coordinates:

$$
\left\{\begin{array}{l}
r=\sqrt{x^{\prime 2}+y^{\prime 2}} \\
\theta=\arctan \left(\frac{y^{\prime}}{x^{\prime}}\right) \\
h=z^{\prime}
\end{array}\right.
$$

\subsection{Projection based pose estimation}

\section{Point tracking}

Given a single point in the 3D volume space $(x, y, z)$, the horizontal and vertical projected coordinate $(u, v)$ of the corresponding volume element or voxel can be uniquely determined given the view transformations $\mathscr{V}$ of the source-volume-detector system. The inverse problem of projection-based point tracking is ill-posed as any point lying on the straight line coming from the source point can cast a shadow on the given detector pixel. Combining $P$ projections from different directions $i$ will overcome this problem and leave you with an overdetermined set of non-linear equations due to the conical shape of the beam.

$$
(u, v)_{i}=\mathscr{V}_{i}(x, y, z) \quad i=1,2, \ldots, P
$$

Using minimally $P=2$ projections, the Levenberg-Marquardt algorithm [16] yields a solution $(x, y, z)_{S}$ that minimizes the sum of squared projection distances. Derivatives of $\mathscr{V}_{i}(x, y, z)$ with respect to changes in $(x, y, z)$ can be calculated analytically.

$$
(x, y, z)_{S}=\underset{x, y, z}{\operatorname{argmin}} \sum_{i=1}^{P}\left|(u, v)_{i}-\mathscr{V}_{i}(x, y, z)\right|^{2}
$$


It is important to notice that this procedure does not optimize the geometry of the scanner system during the optimization. The influence of misalignments in the acquisition system (detector orientation and rotation axis position) may be considered as follows: the vertical $\mathrm{Z}$ axis through the centre of the volume is defined as the rotation axis in the standard reconstruction. Any misalignment is incorporated in the position of the source and the detector by rigid transformations and these effects are additive to the object misalignment. However, the misalignment is constant throughout the acquisition and is typically well-characterized, hence does not affect the measurement of the 3D point coordinates. Furthermore, it should be corrected for in the tomographic reconstruction to avoid negative influence on the reconstruction quality. Additionally, as a result of the general description of this technique, it is not limited to standard circular trajectories. To reach a higher continuous throughput, which is desired in industrial applications, a conveyor belt trajectory [9] could be used.

\section{Axis tracking}

To track the cylinder axis, two 3D points along the axis are sufficient to fix five degrees of freedom, leaving the azimuthal rotation as a last free parameter. In practice these points can be seen by finding the symmetry axis of the cylindrical object on the projections. These points should always correspond to the same height along the cylindrical axis, with a single optimal $(x, y, z)_{S}$ solution to equation 4 . Therefore, segments with a clear boundary at the top are ideal candidates. Due to the symmetric nature of the objects, this algorithm can be executed very fast using tailor-made Graphics Processing Unit (GPU) techniques. This technique of projection-based pose estimation may be impossible for objects of lower symmetry.

The implementation for a specific medical device is illustrated in figure 2(a). First the most stable symmetric feature is segmented. In this device, this segment has a medium projected intensity. To achieve stable results, the darkest regions (i.e. the metal spring, needle and the sample holder below) are first thresholded and dilated. These regions (coloured red) are removed before a higher threshold is set to segment the intermediate intensities. The area that is situated too low in the remaining radiograph (blue) is also subtracted to leave only the edges of the desired segment (green). It is obvious that the specific details of this procedure depend strongly on the object under investigation. As the scope of this work is exactly the inspection of products at the assembly line, this is no real limitation. The same procedure can be used repeatedly once it has been defined and validated.

Now three different techniques are compared to select projected feature points along the symmetry axis:

1. Take the pairwise centres of the four corner pixels at the top and the bottom of the segment;

2. Use minAreaRect function of OpenCV [17] to find the rotated bounding rectangle of the segment with minimal area;

3. Calculate the mean position and the covariance matrix of the segment to fix two points along the axis.

These two centres are tracked on each projection and in this way the 3D position of the object is calculated using equation (4). Each of these techniques will respond differently to grey value fluctuations. The first two are only sensitive to the outer edges, while the third is also subject to variations within the bulk of the material. Note that these projected intensities depend on the spectrum of the beam and the response of the detector. Settings 
that work on one scanner do not necessarily work on other scanners or acquisition setups, so it should be checked if the predefined segmentation values still yield the same results upon implementation at the production site. A realistic polychromatic CT simulation of the complete scanner may be used to predict how different settings balance the reconstructed quality and the accuracy of the pose estimation. Also beam fluctuations at different times of the acquisition process affect the intensity, these can be monitored by selecting an area in the detector that should give a constant intensity (e.g. a rectangle containing only air). Finally, should the segmentation lead to spurious results in a single projection, then outliers can be easily rejected from the tracking algorithm.

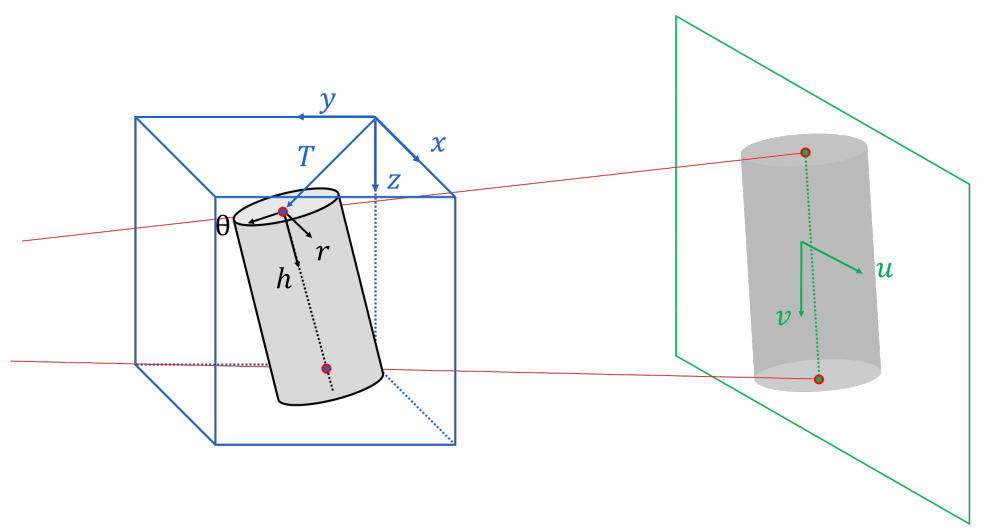

Figure 1: The cylinder can take a free pose in the scanning space. This pose is retrieved by tracking the predefined feature points on projections taken from different directions.

\section{Azimuthal phase}

In some cases it may be necessary to know the azimuthal phase $\gamma$ of the object before reconstructing. When a detailed initial solution or weighted volume is used, any internal rotation of the object around its symmetry axis will produce false features in the reconstruction. In this case, the sample contains only a discrete symmetry in the azimuthal direction with a period of $90^{\circ}$. Since the sample is rotated during the acquisition, this last Euler angle $\gamma$ can be derived by profiling the projected intensity fluctuations in a region parallel to the projected axis. This region is shown as the smaller rectangle in figure 2(a). For all different positions of the sample holder, this intensity profile will give similar results in function of the projection angle, only phase shifted due to the object's internal azimuthal rotation and the geometrical angle $\phi_{G}$ w.r.t. the optical axis of the cone beam scanner.

This perceived phase shift $\phi_{P}$ can be translated back to the last Euler angle when a correction for the first Euler angles $\alpha$ and $\beta$ is also taken into account. After all, the phase was calculated w.r.t. the original $x$-axis, not the $x^{\prime}$-axis.

Although this approach relies on some violation of the continuous cylindrical symmetry of the object, this is not a limitation as it can be noted that the azimuthal phase is only relevant when such violation is present. 


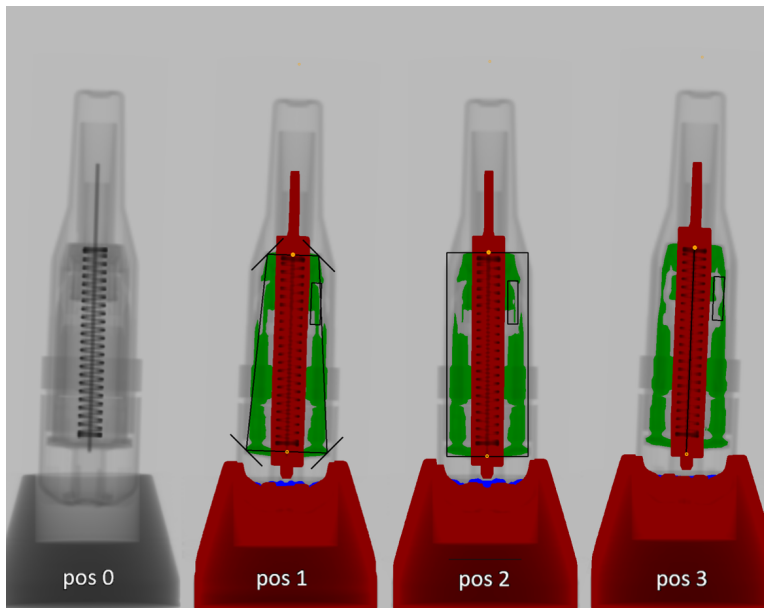

(a)

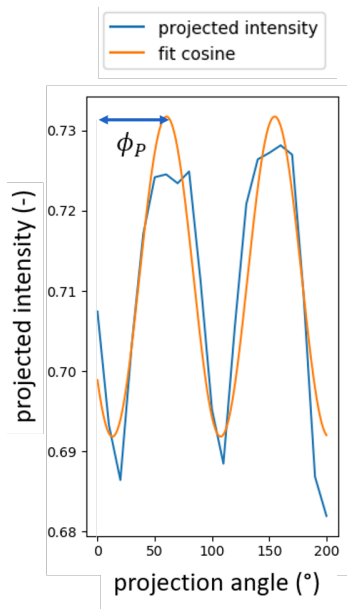

(b)

Figure 2: (a) The projections of a CT acquisition to inspect four medical devices simultaneously are analysed before the reconstruction to estimate the pose of the objects. In position 0 (left), a plain simulated projection of a phantom with known misalignment is shown. Positions 1 to 3 illustrate the three techniques that were compared to select feature points along the axis of the green segments on every position. These are the corner-based technique, the rotated bounding rectangle and the covariance technique. A cosine function was fitted in (b) to the internal fluctuations in the small rectangles of (a) to capture the azimuthal phase of the object. The period of $90^{\circ}$ corresponds to the presence of four repetitions in the azimuthal direction of the object.

$$
\gamma=\phi_{\mathrm{P}}-\phi_{\mathrm{G}}-\arctan \left(\frac{\tan (\alpha)}{\cos (\beta)}\right)
$$

\section{Accuracy and precision of the pose estimation}

The pose estimation accuracy is crucial information when reconstructing objects with periodic cylindrical symmetry using an initial volume. Any mismatch of the pose counteracts the advantages of using an initial volume, and impedes fast inspection analyses that rely on exact spatial coordinates.

To test the reliability of the projection-based pose estimation, a scan of the test object was simulated using a CAD-designed model. Such models are often available in manufacturing industry, although it should be noted that these usually represent an ideal sample without any defects. During the production, deviations from this ideal sample will appear. These deviations are in this case exactly the interest of the $\mathrm{CT}$ inspection, but they may also affect the outcome of the pose estimation. This influence should be kept to a minimum by selecting the most stable components, if possible.

This reliability was measured by simulating projections of a single CAD model of the device at 600 different poses in total at four different lateral positions of the rotation stage. The 
poses and linear attenuation coefficients were derived from a real scan with the same geometry, to ensure that they resemble actual situations. The projection simulator that was used here is the same simulator that was used in the iterative reconstruction introduced in section 2.2 The difficulty of this approach is that the linear attenuation coefficients of the phantom should match those of a standard CT scan on the same setup to yield the same projected intensities. To avoid this step, a polychromatic projection simulator could be used where the linear attenuation coefficients are replaced by more relevant material properties (i.e. chemical composition and density) [18].

The simulated geometry resembles an in-line CT inspection setup (see figure 2(a)). As can be seen in the scanner settings in table 1 the objects are placed rather close to the wide detector in order to scan more samples at the same time. The rotation stage is a combination of four holders that rotate at the same speed. The distance from the source to the object was calibrated by scanning a phantom with known dimensions. Furthermore, the detector misalignment and the projected horizontal coordinate of the rotation axis, which influence the reconstruction quality, are also assumed to be stable over multiple scans.

\subsection{SART reconstruction in cylindrical coordinate system}

In order to situate which extensions have been made to incorporate the cylindrical coordinate system into the iterative reconstruction framework as previously described in [19, 20], a brief overview is given in this section. CT reconstruction seeks to calculate the distribution of attenuation coefficients $\mu$ of an object given the projected X-ray intensities on the detector for a number of projection angles. The basic operation in these methods is back-projection, which tries to invert the set of monochromatic Lambert-Beer equations for all pixel values $p_{j}$ to a grid of attenuation coefficients $\mu_{l}$ :

$$
p_{j}=-\ln \left[\frac{I_{j}}{I_{0, j}}\right]=\sum_{l=0}^{L} t_{j l} \cdot \mu_{l}
$$

The weights $t_{j l}$ represent the intersection length of the ray $j$ passing through the voxel $l$, where $j$ runs over all pixels of all projection images. In the SART approach, the solution is found by updating each voxel with a correction factor that is found by comparing the actual projection with a simulation on the current state of the volume [19]. This update step $k$ is performed for each projection in SART. The correction factor is accompanied by the relaxation $\lambda$ which dampens the contribution to each update.

$$
\vec{\mu}_{m}^{(k+1)}=\vec{\mu}_{m}^{(k)}+\lambda \sum_{j \in \mathrm{OS}} \frac{\left(p_{j}-\sum_{l=0}^{L} t_{j l} \cdot \mu_{l}^{(k)}\right)}{\sum_{l=0}^{L} t_{j l}} \cdot \frac{t_{j m}}{\sum_{j \in \mathrm{OS}} t_{j m}}
$$

The ordered subset (OS), which groups radiographies for each update step, corresponds to one full image in SART. The size of the ordered subset can be increased to trade off convergence speed for more stability. The projection step is calculated in a pixel driven sampling approach, by tracing the rays between source and detector pixel with an equidistant sampling scheme in Cartesian space. The ray-voxel intersection weights $t_{j l}$ are calculated intrinsically as the number of samplings in each voxel. The extension added in this work entails that the attenuation coefficients are interpolated on the aligned cylindrical grid. This method is also easily extendable to other division schemes. 
The same extension can be used for the back-projection step, which is executed in a voxel driven approach. Only the coordinate transform (2) from the cylindrical to the original Cartesian coordinates is applied before the corresponding correction term is accessed. The total overhead translates in this case to one cylindrical transformation each time a volume access is performed. The alignment matrix needs to be precomputed for equation (1).

Weighted back-projection, as implemented by Heyndrickx et al. [20], uses a volume with weights to describe the likelihood of change for every voxel in the reconstruction. The correction term in equation 7 contained an extra factor $w_{m} / W_{j}$, where the normalization factors are computed by integration of the $L$ weights along the straight line to the pixel $j$ :

$$
W_{j}=\frac{\sum_{l=0}^{L} t_{j l} \cdot w_{l}}{\sum_{l=0}^{L} t_{j l}}
$$

The weights $w$ are assigned as a function of the attenuation values in the initial volume: $w=1+v \cdot \exp \left(-\mu^{2} /\left(2 \sigma^{2}\right)\right)$, in this work using $v=4, \mu=0.45 \mathrm{~cm}^{-1}, \sigma=0.1 \mathrm{~cm}^{-1}$. The weight volume can be similarly described in the cylindrical coordinate system, without affecting the core of the algorithm.

\subsection{MTF measurements on phantom data}

Due to the way that the reconstruction grid is divided, the number of azimuthal divisions can be changed effortlessly. Depending on the application, reducing the number of voxels in a direction that contains broad, smooth structures can be tolerated or may even be necessary to reduce aliasing effects.

To get a sense of the resolution dependence on the number of azimuthal voxels $\left(N_{\theta}\right)$ and number of radial voxels $\left(N_{r}\right)$, we perform a high detail simulation on samples consisting of radial or azimuthal lines as shown at the left in figure 3 The simulations are made with an excessive amount of 4096 projections with a width of 2048 pixels, to ensure this projection does not affect the reconstruction. In the four reconstructions on the right, a deviation can be observed especially for smaller radial positions. This is only a single example, with clear visual effects, that was selected to illustrate how these phantoms are used to quantitatively measure the response of the cylindrical reconstruction.

The directional Modulation Transfer Function (MTF) [21] (radial or azimuthal MTF) of the reconstruction is determined by measuring the modulation (resp. $m_{r}$ and $m_{\theta}$ ) for varying frequency factor, i.e. the number of lines $n_{l}$ in the phantom of constant size. The modulation $m\left(n_{l}\right)$ for a given frequency $n_{l}$ is determined by measuring the minimum and maximum reconstructed attenuation coefficients $\mu_{\min }$ and $\mu_{\max }$ in the central slice (to eliminate the effect of cone beam artefacts).

$$
m\left(n_{l}\right)=\frac{\mu_{\max }\left(n_{l}\right)-\mu_{\min }\left(n_{l}\right)}{\mu_{\max }\left(n_{l}\right)+\mu_{\min }\left(n_{l}\right)}
$$

This value varies between 0 and 1 , given the positive values of $\mu$. A value of 1 is expected to be the result of a good response of the reconstruction algorithm to the used phantom frequency. However, one downside to this measure is that it does not punish aliasing effects that alter the line frequency but maintain the modulation depth. A visual inspection is also 
required to investigate these faulty cases. In all reconstructions, also the radial dependency of the MTF is measured. For the azimuthal MTF $\left(m_{\theta}\right)$ this is simply the MTF at each $r$. The radial dependency of the radial MTF $\left(m_{r}\right)$ is calculated with a short-range sliding window over the line period. The window width is equal to one period of the phantom. The slide step is equal to the width instead of 1 voxel, for ease of implementation.

The lines are modelled by a cosine function on the respective direction. For the $m_{\theta}$, each horizontal slice (in Cartesian coordinates) of the phantom looks like a Siemens star pattern [22]. The $m_{r}$ is measured in a phantom of concentric circles. Highly detailed projections are simulated on these samples of shape $\left(S_{h}, S_{\theta}, S_{r}\right)=(32,1608,256)$. The frequencies are doubled until a factor of $128\left(=S_{r} / 2\right)$ is reached. The image is projected on 4096 images of dimensions $(W, H)=(2048,128)$ to reduce the influence of sampling artefacts in the simulation step.
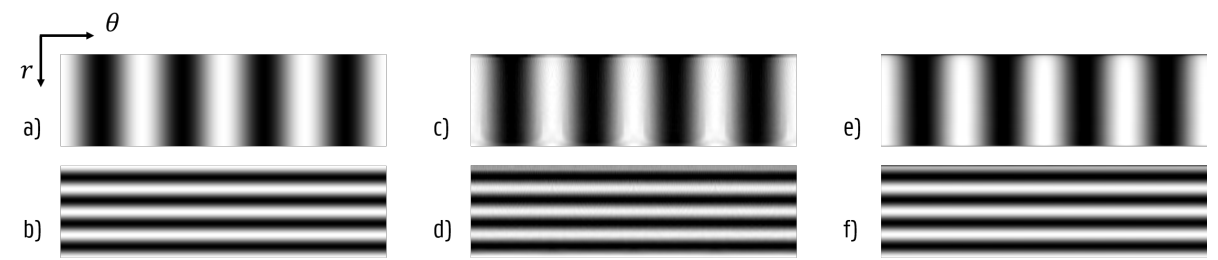

Figure 3: Azimuthal (a) and radial (b) line phantoms, here with $n_{l}=4$, are created to test the response of the cylindrical reconstruction method. In (c) and (d), the full-resolution projections of respectively (a) and (b) are reconstructed on cylindrical slices with $\left(N_{\theta}, N_{r}\right)=$ $(1608,32)$. Analogously, (e) and (f) show azimuthally sub-sampled reconstructions with $\left(N_{\theta}, N_{r}\right)=(32,256)$. The reconstructions are quantitatively assessed by the $m_{\theta}$ (top) and the $m_{r}$ (bottom).

\subsection{Application in batch CT scan inspection}

To illustrate how the developed strategy can be applied in a real production environment, a scan was performed on a series of medical devices with high safety demands. 50 objects have been scanned, reconstructed and analysed for artificially induced internal defects. The analysis of the devices is beyond the scope of this article. Only one test outcome, measuring the presence of a particular piece, will be presented to assess the influence of the different reconstruction strategies:

(a) The object is reconstructed without aid of initial volume or weighted back-projection.

(b) Include an initial solution, which is considered to be the dangerous approach in the case of missing components. The iterative algorithm might not remove these sufficiently.

(c) Only weighted back-projection: the algorithm starts from a completely empty volume.

(d) Initial solution combined with weighted back-projection. Some predefined regions are given higher weights to change their attenuation value. 
Table 1: Imaging parameters for high speed circular cone beam scans of medical devices.

\begin{tabular}{|llcrc|}
\hline Geometry & Circular cone beam & & 791 & $\mathrm{~mm}$ \\
& Source Detector Distance & SDD & 679 & $\mathrm{~mm}$ \\
& Source Object Distance & SOD & 21 & \\
& Number of projections & $N_{p}$ & $200^{\circ}$ & \\
& Last scan angle & & & \\
Detector & Varex 1512 & & & \\
& Detector size & $(\mathrm{H}, \mathrm{W})$ & $(1536,1944)$ & \\
& Detector pixel size & $p_{s}$ & 0.0748 & $\mathrm{~mm}$ \\
& Projected pixel size & $p_{v}$ & 0.06 & $\mathrm{~mm}$ \\
Source mode & Exposure time & & $130 \times 9$ & $\mathrm{~ms}$ \\
& Large focus & & 60 & $\mathrm{kV}$ \\
& Voltage & & 18 & $\mathrm{~W}$ \\
Sample & Power & & & \\
& KN-S & & 64 & $\mathrm{~mm}$ \\
& Height & & 8 & $\mathrm{~mm}$ \\
\hline
\end{tabular}

\section{Results}

3.1 Characterization of cylindrical reconstruction on simulated data

The Modulation Transfer Function was used to quantify the response of the cylindrical reconstruction on phantom samples with varying spatial frequency in the azimuthal or radial direction. This test was performed to give insight into the following aspects of the reconstruction:

1. What is the behaviour at low radii, where the azimuthal voxel dimensions are smaller?

2. How does downsampling the cylindrical coordinate system affect the signal modulation?

3. What is the influence of CT acquisitions with angular sub-sampling?

The $m_{\theta}$ and $m_{r}$ at reconstruction shape $\left(N_{h}, N_{\theta}, N_{r}\right)=(32,512,256)$ are shown in figure 4. As expected, the MTF becomes worse at high frequent lines. The azimuthal MTF shows worse behaviour at small radii. Even though the voxel size is virtually decreased at lower $r$, the actual resolution does not automatically follow this trend. For the radial MTF, only a minor effect of the centre distance can be observed in figure 4 (b).

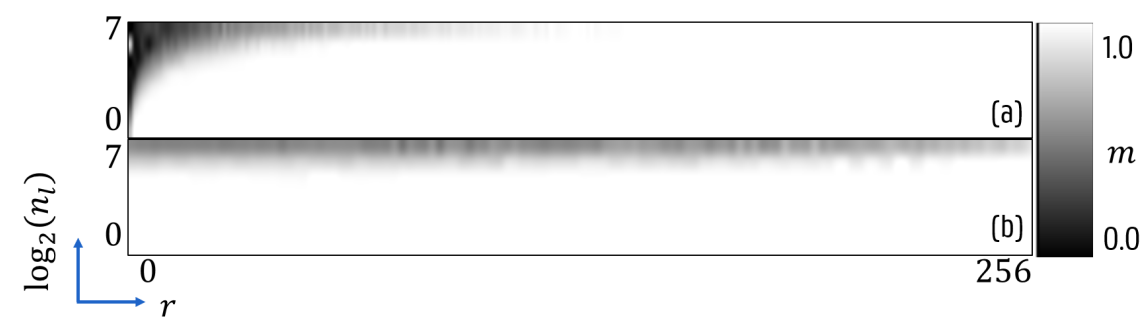

Figure 4: At each radial position $r$ of the reconstruction, the $m_{\theta}$ (a) and $m_{r}$ (b) are measured. Each vertical line represents an MTF. The $m_{\theta}$ (measured in the Siemens star-like phantom), becomes worse at low radii, not being able to reconstruct the high frequency signals. For the $m_{r}$, only a small radial dependence is found. 
No variation of the $m_{r}$ was noted for any $N_{\theta}$ between 1 and 512. Note that this is a very extreme phantom and the results will probably be a mixed term in real objects.

The same exercise has been done over for a lower number of projections $\left(N_{p}\right)$. Now 67 instead of 4096 projections were simulated and the same reconstruction shape was used. Similar results have been noted for 64 projections, the odd number of 67 was chosen to avoid any numerical bias. These results are shown in figure 5 and the modulation factor is lower than the previous outcome as expected. More variation can be observed for the $m_{r}$ phantom in the radial direction. When we look at the reconstructed slices in figure 6 , the origin of the seemingly good $m_{r}$ at high $r$ is revealed. Due to the insufficient amount of projections, aliasing effects appear in the reconstruction. Actually, the quality is better at low $r$ where no azimuthal aliasing shows up at lower frequencies. Decreasing the number of azimuthal divisions $N_{\theta}$ to the order of the projections number gives more truthful results, though the aliasing is still visible. When the same radial resolution is required at a greater distance from the central axis, a higher $N_{p}$ is required. This requirement remains true for hollow cylinders. Understanding these aliasing effects can help in the development of more advanced sampling strategies.

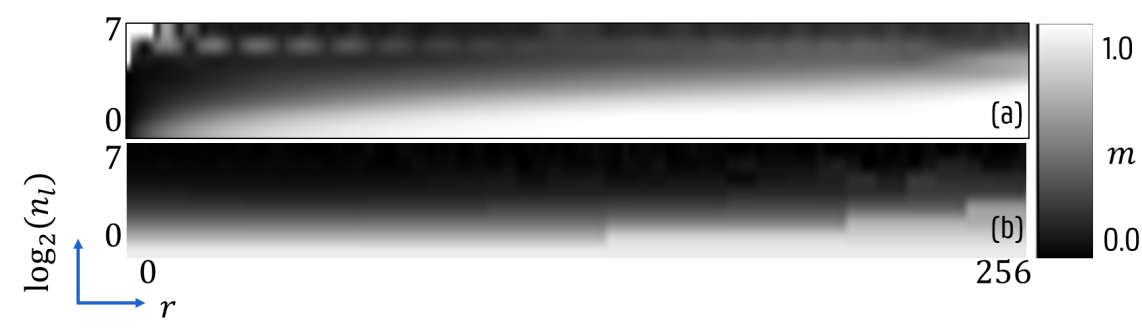

Figure 5: When fewer projections are taken, both $m_{\theta}$ (a) and $m_{r}$ (b) are affected, but they behave similarly to the previous case (figure 4). The white pattern in the top image, which indicates a seemingly high $m_{\theta}$ at high frequencies, is most likely caused by reconstruction aliasing effects where the MTF method fails.

\subsection{Accuracy and precision of the pose estimation}

The accuracy and precision of the projection-based pose estimation using the three different feature selection techniques (listed in section 2.1- Axis tracking) are given in tables 2, 3 . 4 respectively. For these scans, the axis detection using the first feature selection technique (using extremal corner pixels) took only $0.23 \mathrm{~s}$ on average. The two other techniques (using rotated bounding rectangles or the covariance approach) were slightly slower with $0.35 \mathrm{~s}$ each. Although these timings strongly depend on the used hardware, the relative increase will probably be similar.

The measured poses at four different positions of the rotation stage were compared to remark any systematic deviation. The deviations in the pose of the reconstructed object and the phantom object were captured using the differences of four observed quantities. 

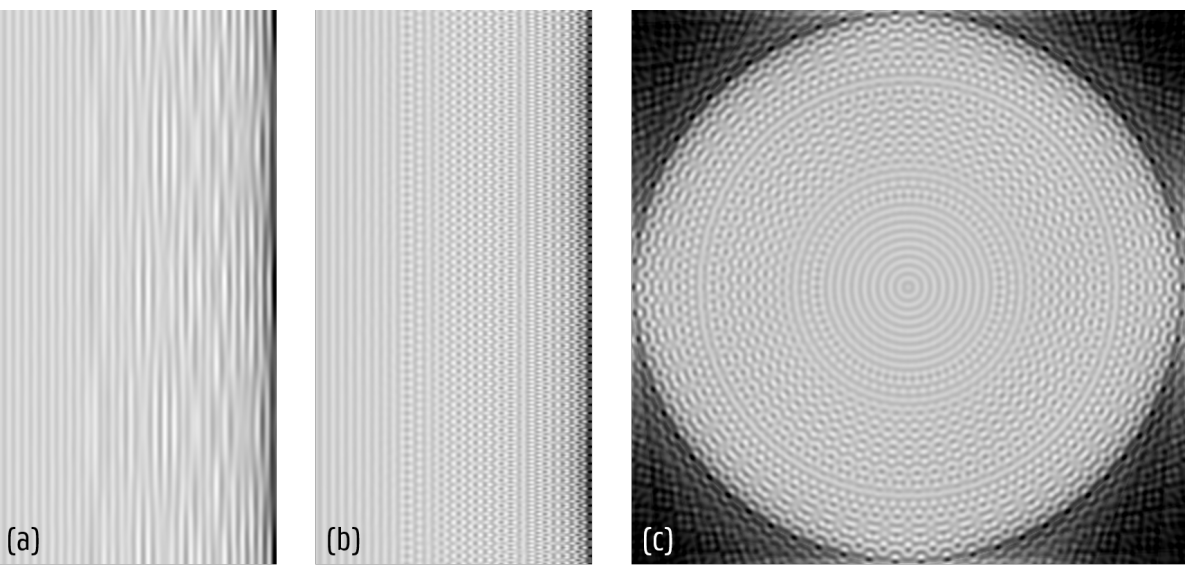

Figure 6: Decreasing $N_{\theta}$ in the $r \theta$ slice (a) compared to (b) reduces aliasing artefacts in the azimuthal direction when an insufficient amount of projections is given. This cannot be done for a Cartesian reconstruction (c), where the results are similar to the reconstruction in (b).

Table 2: Measured accuracy and precision for the projection-based pose estimation using the corner-based approach.

\begin{tabular}{|rr|rrrrr|}
\hline & pose deviation & overall & pos 0 & pos 1 & pos 2 & pos 3 \\
\hline & azimuthal angular accuracy $\left(^{\circ}\right)$ & -4.402 & -2.984 & -4.325 & -4.682 & -5.617 \\
& precision $\left(^{\circ}\right)$ & 4.149 & 2.927 & 2.436 & 4.712 & 5.346 \\
\hline \multirow{2}{*}{ top } & transverse accuracy $(\mathrm{mm})$ & 0.077 & 0.077 & 0.085 & 0.073 & 0.072 \\
& precision $(\mathrm{mm})$ & 0.033 & 0.033 & 0.032 & 0.033 & 0.033 \\
\hline \multirow{2}{*}{ middle } & transverse accuracy $(\mathrm{mm})$ & 0.060 & 0.064 & 0.067 & 0.057 & 0.051 \\
& precision $(\mathrm{mm})$ & 0.025 & 0.025 & 0.024 & 0.025 & 0.024 \\
\hline \multirow{2}{*}{ bottom } & transverse accuracy $(\mathrm{mm})$ & 0.050 & 0.060 & 0.054 & 0.049 & 0.037 \\
& precision $(\mathrm{mm})$ & 0.020 & 0.019 & 0.019 & 0.019 & 0.017 \\
\hline
\end{tabular}

These quantities are the internal azimuthal angles and the locations of the axis top, middle (31.2 $\mathrm{mm}$ from the top along the axis) and bottom (62.4 $\mathrm{mm}$ along the axis).

From this table, it can be seen that the internal azimuthal angle is known with a precision of $4.2^{\circ}$. The systematic deviation of the internal angle of $-4.4^{\circ}$ could be contributed to the naive assumption that the maximal attenuation should correspond to a perpendicular position of the internal structure. This systematic deviation can be easily corrected by subtracting this value from the pose estimation. A small increasing deviation between the results from the different rotation axis positions could be found.

The overall transverse accuracy of the pose is best at the bottom of the sample, where the device is contained by the sample holder. The results are comparable to the projected pixel size of $0.06 \mathrm{~mm}$. For these pose estimations, 21 projections were used to trace back the positions along the axis. The longitudinal precision is nearly constant at $0.011 \mathrm{~mm}, 0.031 \mathrm{~mm}$ and $0.025 \mathrm{~mm}$, for each feature selection technique respectively. Constant systematic deviations along the axis can be compensated by defining the origin of the cylindrical coordinates higher or lower along the axis. 
Table 3: Measured accuracy and precision for the projection-based pose estimation using the rotated bounding rectangle approach.

\begin{tabular}{|rr|rrrrr|}
\hline & pose deviation & overall & pos 0 & pos 1 & pos 2 & pos 3 \\
\hline & ${\text { azimuthal angular accuracy }\left(^{\circ}\right)}^{\circ}$ & -0.961 & -0.362 & -0.659 & -1.492 & -1.330 \\
& ${\text { precision }\left({ }^{\circ}\right)}^{*}$ & 13.350 & 12.438 & 11.517 & 15.658 & 13.397 \\
\hline \multirow{2}{*}{ top } & transverse accuracy $(\mathrm{mm})^{\text {middle }}$ & 0.362 & 0.416 & 0.383 & 0.302 & 0.347 \\
& precision $(\mathrm{mm})$ & 0.164 & 0.176 & 0.177 & 0.113 & 0.159 \\
\hline bottom & transverse accuracy $(\mathrm{mm})$ & 0.186 & 0.215 & 0.203 & 0.159 & 0.166 \\
& precision $(\mathrm{mm})$ & 0.081 & 0.087 & 0.085 & 0.061 & 0.076 \\
& transverse accuracy $(\mathrm{mm})$ & 0.067 & 0.060 & 0.071 & 0.071 & 0.064 \\
& precision $(\mathrm{mm})$ & 0.025 & 0.024 & 0.026 & 0.022 & 0.025 \\
\hline
\end{tabular}

Table 4: Measured accuracy and precision for the projection-based pose estimation using the covariance approach.

\begin{tabular}{|rr|rrrrr|}
\hline & pose deviation & overall & pos 0 & pos 1 & pos 2 & pos 3 \\
\hline & azimuthal angular accuracy $\left(^{\circ}\right)$ & -5.993 & -5.975 & -6.664 & -3.735 & -7.596 \\
& precision $\left({ }^{\circ}\right)$ & 19.579 & 17.497 & 16.842 & 22.707 & 20.488 \\
\hline \multirow{2}{*}{ top } & transverse accuracy $(\mathrm{mm})$ & 0.236 & 0.227 & 0.248 & 0.240 & 0.229 \\
& precision $(\mathrm{mm})$ & 0.094 & 0.084 & 0.089 & 0.095 & 0.105 \\
\hline \multirow{2}{*}{ middle } & transverse accuracy $(\mathrm{mm})$ & 0.082 & 0.085 & 0.091 & 0.078 & 0.072 \\
& precision $(\mathrm{mm})$ & 0.040 & 0.037 & 0.039 & 0.040 & 0.039 \\
\hline bottom & transverse accuracy $(\mathrm{mm})$ & 0.108 & 0.098 & 0.099 & 0.115 & 0.118 \\
& precision $(\mathrm{mm})$ & 0.058 & 0.053 & 0.052 & 0.059 & 0.063 \\
\hline
\end{tabular}

3.3 Use of initial volume and back-projection weights in cylindrical reconstruction algorithms

The added value of the initial solution and the weighted back-projection are investigated for the batch scan with settings listed in table 1 . The number of voxels in the cylindrical reconstruction is $\left(N_{h}, N_{\theta}, N_{r}\right)=(240,40,60)$ for a virtual voxel size of $0.312 \mathrm{~mm}$. Reconstruction times changed from $0.30 \mathrm{~s}$ for a normal one, to $0.52 \mathrm{~s}$ for a weighted back-projection reconstruction. There is virtually no overhead for the reconstruction using an initial volume, since this can be stored in memory during the batch reconstruction. In figure 7, the results are illustrated for each distinct reconstruction strategy described in section 2.4 .

From figure 7, a number of preliminary conclusions can be derived. When no prior info is included, the slices have low contrast and sharpness. This will negatively affect the later analysis phase where image characteristics are converted to binary results (present / missing). In sub-figure 7(b) a higher sharpness is perceived compared to figure 77a). For this reconstruction, the initial volume as seen in figure $7 \mathrm{f}$ ) was used. This volume should not necessarily be equal to the ideal picture that is given in figure 7 (e). In this initial volume, the spring component (the bright, vertically extended ellipse on cylindrical slice) was averaged over all azimuthal voxels, to incorporate that this component was able to move freely in this direction. The weighted approach in figure 7 (d) removes the blurred streaks from the static air regions. Remark that the noise contribution in the dynamic regions of the reconstruction using weighted back-projection is higher than the standard approach that uses equal contributions in the back-projection step. This is particularly true for the reconstruction in 

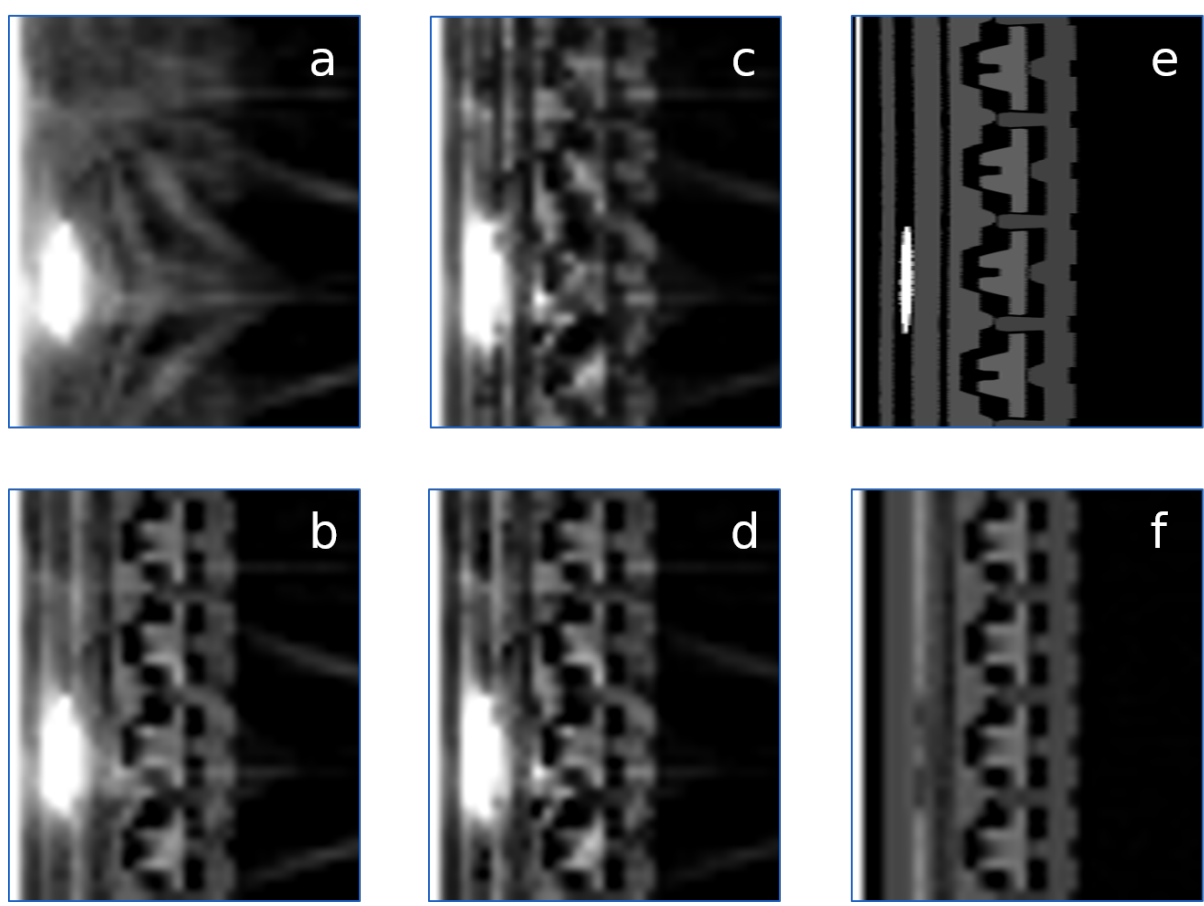

Figure 7: A cropped $r \theta$ slice of four different cylindrical reconstructions, severely influenced by a dense metal spring and needle on the left of each slice. (e) shows the phantom that was used. The initial volume in (f) only includes structure in the azimuthal direction $\theta$ where the components can be fixed. In (a), the SART reconstruction using 21 projections is executed without prior knowledge. (b) and (d) use an initial volume, while weighted back-projection is applied in (c) and (d).

figure 7(c) that does not include an initial solution. However, the response to anomalous cases where specific components are missing from the sample is expected to be better using weighted back-projection.

The results can also be observed in the inspection test measuring the presence of a particular piece, as described above in 2.4 Figure 8 shows the distribution of this measure for the different methods. It is clear that the difference between the groups has increased most when an initial volume and weights are included. This clear distinction is necessary to increase the detection rate of the internal defects without discarding safe devices.

\section{Conclusion}

In this work two techniques have been developed in the scope of high-throughput CT inspection of cylindrical objects. These are necessary to reduce scan time while maintaining reasonable image quality. A pose estimation technique for cylindrical objects has been implemented that uses only basic segmentation algorithms on the projected images. The axis extraction using the corner information was found to be both fast and precise. Reconstruc- 


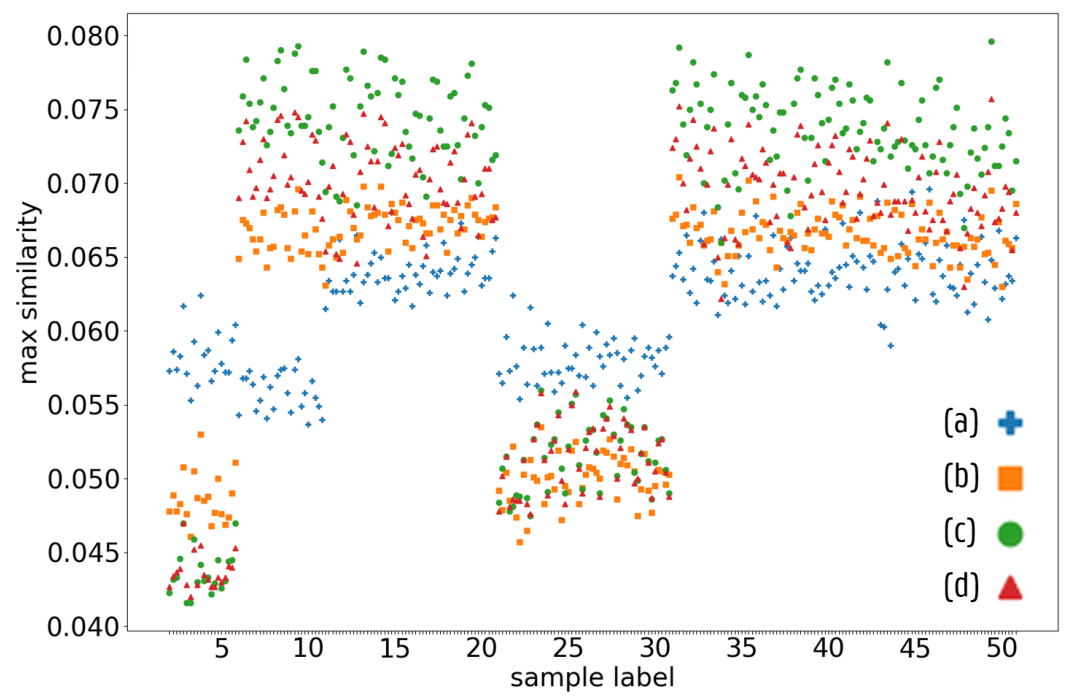

Figure 8: When the reconstruction contrast is improved, it becomes easier to spot a missing component in samples (0-5) and (20-30). The labels (a-d) correspond to the ones given in figure 7

tion was performed directly in the aligned cylindrical coordinate system.

The MTF characterization shows how reliably the scanner can reconstruct different frequencies of the (phantom) object. The artificially high number of azimuthal divisions at low radius should only be interpreted as a mathematical construct. The actual resolution corresponds more to the Cartesian voxel size. The number of divisions could be matched better with the actual content of the object. When the components only have a low frequency in the azimuthal direction, the number of divisions may be reduced. For the radial direction, a similar reasoning applies. This reduces the reconstruction time.

The aligned reconstruction allows the inclusion of more advanced techniques when a series of same-type objects is scanned. An initial volume can be used to achieve faster convergence. Weighted reconstruction adds a higher sensitivity to changes.

The methods work for scans of cylindrical objects and can be easily added to existing frameworks. The SART reconstruction technique is not mandatory, any iterative method can benefit from alignment. The coordinate change yields equal reconstruction quality in the most simple case. The adaptation to the symmetry of the objects allows that more advanced techniques are added to improve image quality. 


\section{Acknowledgements}

This research is funded by the imec ICON project iXCon (Agentschap Innoveren en Ondernemen project nr. HBC.2016.0164). The work by M. Heyndrickx was funded by the Research Foundation Flanders (FWO) [grant number 1S 215 16N]. The Ghent University Special Research Fund (BOF-UGent) is recognized for the financial support of the Centre of Expertise UGCT (BOF.EXP.2017.0007).

The authors would like to thank the manufacturer for providing the samples with introduced faults and also TESCAN XRE for acquiring the projection data (www.tescan. com). Jan Aelterman is also acknowledged for proofreading the submitted manuscript.

\section{References}

1. R. P. Haff, N. Toyofuku, X-ray detection of defects and contaminants in the food industry, Sensing and Instrumentation for Food Quality and Safety 2 (4) (2008) 262-273.

2. Y. Zou, D. Du, B. Chang, L. Ji, J. Pan, Automatic weld defect detection method based on Kalman filtering for real-time radiographic inspection of spiral pipe, NDT \& E International 72 (2015) 1-9.

3. J. Xu, T. Liu, X. Yin, B. Wong, S. B. Hassan, Automatic X-ray crack inspection for aircraft wing fastener holes, in: 2nd International Symposium on NDT in Aerospace, Vol. 1, 2010.

4. J. Duvillier, M. Dierick, J. Dhaene, D. Van Loo, B. Masschaele, R. Geurts, L. Van Hoorebeke, M. N. Boone, Inline multi-material identification via dual energy radiographic measurements, NDT \& E International 94 (2018) 120-125.

5. J. P. Kruth, M. Bartscher, S. Carmignato, R. Schmitt, L. De Chiffre, A. Weckenmann, Computed tomography for dimensional metrology, CIRP Annals-Manufacturing Technology 60 (2) (2011) 821-842.

6. E. Ametova, M. Ferrucci, S. Chilingaryan, W. Dewulf, A computationally inexpensive model for estimating dimensional measurement uncertainty due to X-ray computed tomography instrument misalignments, Measurement Science and Technology 29 (6) (2018) 065007.

7. G. Jones, P. Huthwaite, Limited view X-ray tomography for dimensional measurements, NDT \& E International 93 (2018) 98-109.

8. E. Shefer, A. Altman, R. Behling, R. Goshen, L. Gregorian, Y. Roterman, I. Uman, N. Wainer, Y. Yagil, O. Zarchin, State of the art of CT detectors and sources: a literature review, Current Radiology Reports 1 (1) (2013) 76-91.

9. T. De Schryver, J. Dhaene, M. Dierick, M. N. Boone, E. Janssens, J. Sijbers, M. van Dael, P. Verboven, B. Nicolai, L. Van Hoorebeke, In-line NDT with X-ray CT combining sample rotation and translation, NDT \& E International 84 (2016) 89-98.

10. K. J. Batenburg, J. Sijbers, Dart: a practical reconstruction algorithm for discrete tomography, IEEE Transactions on Image Processing 20 (9) (2011) 2542-2553.

11. E. Janssens, J. De Beenhouwer, M. Van Dael, T. De Schryver, L. Van Hoorebeke, P. Verboven, B. Nicolai, J. Sijbers, Neural network Hilbert transform based filtered backprojection for fast inline X-ray inspection, Measurement Science and Technology 29 (3) (2018) 034012.

12. T. Christian, L. Jean-Daniel, F. Réjean, L. Roger, Fully 3d iterative CT reconstruction using polar coordinates, Medical Physics 40 (11) (2013) 111904. 
13. L. Jian, L. Litao, C. Peng, S. Qi, W. Zhifang, Rotating polar-coordinate ART applied in industrial CT image reconstruction, NDT\&E International 40 (4) (2007) 333 - 336.

14. M. Bui, S. Albarqouni, M. Schrapp, N. Navab, S. Ilic, X-ray PoseNet: 6 DoF pose estimation for mobile X-ray devices, in: 2017 IEEE Winter Conference on Applications of Computer Vision (WACV), IEEE, 2017, pp. 1036-1044.

15. A. Presenti, S. Bazrafkan, J. Sijbers, J. De Beenhouwer, Deep learning-based 2D-3D sample pose estimation for X-ray 3DCT, in: 10th Conference on Industrial Computed Tomography (iCT) 2020, NDT \& E International, 2020.

16. D. W. Marquardt, An algorithm for least-squares estimation of nonlinear parameters, Journal of the Society for Industrial and Applied Mathematics 11 (2) (1963) 431-441.

17. Itseez, Open source computer vision library, https://github.com/itseez/opencv (2015).

18. J. Dhaene, E. Pauwels, T. De Schryver, A. De Muynck, M. Dierick, L. Van Hoorebeke, A realistic projection simulator for laboratory based X-ray micro-CT, Nuclear Instruments and Methods in Physics Research Section B: Beam Interactions with Materials and Atoms 342 (2015) 170-178.

19. T. De Schryver, Fast imaging in non-standard X-ray computed tomography geometries, Ph.D. thesis, Ghent University (2017).

20. M. Heyndrickx, T. De Schryver, M. Dierick, M. Boone, T. Bultreys, V. Cnudde, L. Van Hoorebeke, Improving the reconstruction of dynamic processes by including prior knowledge, in: HD-Tomo-Days, Abstracts, 2016, p. 1.

21. G. D. Boreman, Modulation transfer function in optical and electro-optical systems, Vol. 21, SPIE press Bellingham, WA, 2001.

22. A. González-López, C. Ruiz-Morales, MTF determination from a star bar pattern image, Medical physics 42 (9) (2015) 5060-5065. 